\section{Características clínicas y pronóstico de pacientes con cáncer de mama HER2 positivo avanzado, en la era antes y después de terapias anti-HER2}

\author{
CESAR SÁNCHEZ ${ }^{1}$, FRANCISCO DOMÍNGUEZ ${ }^{2}$, \\ HÉCTOR GALINDO ${ }^{1}$, MAURICIO CAMUS ${ }^{2}$, DAVID ODDÓ ${ }^{3}$, \\ ALEJANDRA VILLARROEL ${ }^{3}$, DRAVNA RAZMILIC, \\ MARÍA ELENA NAVARRO ${ }^{4}$, ALEJANDRA PÉREZ-SEPÚLVEDA ${ }^{1}$, \\ LIDIA MEDINA ${ }^{5, a}$, VALESKA LÓPEZ $Z^{5, a}$, FRANCISCO ACEVEDO ${ }^{1}$
}

\section{Survival of patients with advanced HER2+ breast cancer. Analysis of a cancer center database}

Background: HER2+ breast cancer (BC) subtype overexpresses the Human Epidermal growth factor Receptor type-2 (HER2) and is characterized by its aggressiveness and its high sensitivity to monoclonal antibody-based HER2-targeted therapies. Aim: To assess the prognosis and evaluate the impact of novel anti-HER 2 therapies on advanced HER $2+B C$ patients treated at our institution over the last decades. Material and Methods: Analysis of the patient database at a cancer center of a university hospital. Information about the subtype of cancer was obtained in 2,149 of 2,724 patients in the database. Eighteen percent of the latter were HER2+. We analyzed data of 83 of these patients with advanced disease. Results: Median overall survival (OS) was 24 months. For patients treated between 1997-2006 median OS was 17 months and for those treated in the period 2007-2017 median OS was 32 months ( $p=0.09$ ). Conclusions: $A$ non-significant trend towards better survival in the last decade was observed. HER2+BC overall survival has improved in our center. This can be probably attributed to the use of novel more effective anti-HER2 therapies.

(Rev Med Chile 2018; 146: 1095-1101)

Key words: Breast Neoplasms; Survival; Trastuzumab.

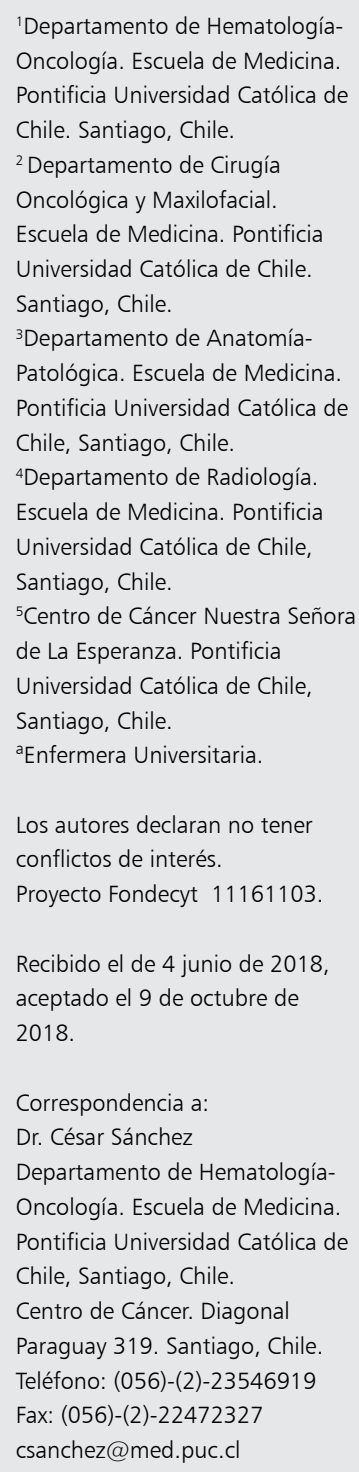

E 1 cáncer de mama $(\mathrm{CM})$ es la primera causa de muerte en mujeres chilenas ${ }^{1}$, y su pronóstico depende principalmente de la extensión o etapa de la enfermedad y del subtipo tumoral ${ }^{2}$. El 5\% de las pacientes con CM se presenta con enfermedad en etapa avanzada al diagnóstico y hasta $20-30 \%$ de las pacientes con enfermedad localizada puede sufrir recurrencias de la enfermedad durante el seguimiento ${ }^{3,4}$.

Mientras pacientes con CM localizado logran tasas de sobrevida global (SG) a 5 años mayores a $90 \%$, la SG a 5 años en pacientes con CM avanzado es cercana a $20 \%{ }^{4}$.

La determinación del subtipo del CM permite evaluar su historia natural y determinar tratamientos específicos. El subtipo HER2+ (con sobreexpresión del receptor del factor de crecimiento epidérmico tipo 2 [HER2] o amplificación de su gen) explica hasta $25 \%$ de los CM, y se caracteriza por una evolución agresiva y peor pronóstico en 
ausencia de tratamiento ${ }^{5}$. En este subtipo, el manejo en base a quimioterapia y agentes anti-HER2 ha cambiado su evolución, mejorando la sobrevida libre de enfermedad, así como la SG ${ }^{6}$.

La incorporación de agentes anti-HER2 en guías clínicas y mejor acceso a su uso en nuestro país (gracias a la ley 20.850, ley "Ricarte Soto", promulgada el 1 de junio de 2015) ha aumentado su aplicación en el escenario de CM HER2+ avanzado ${ }^{7}$. Para conocer el impacto de estos tratamientos en la SG de pacientes con CM avanzado HER2+ en nuestra realidad clínica, analizamos la experiencia y resultados clínicos de pacientes con CM HER2+ avanzado, tratados en nuestro centro, en las últimas dos décadas.

\section{Metodología}

Estudio retrospectivo, descriptivo, aprobado por el comité ético científico de nuestra institución.

Analizamos nuestra base de datos de pacientes con CM, tratados en el Centro de Cáncer Nuestra Señora de La Esperanza de la Pontificia Universidad Católica de Chile y Red de Salud UC-Christus, desde el año 1997 hasta septiembre del año 2017. Se seleccionó pacientes con CM HER2+ avanzado (luminal B o HER2 enriquecido), definido como aquellos pacientes que debutaron con enfermedad metastásica y los que recurrieron con enfermedad sistémica o inoperable.

Las características clínicas analizadas fueron: edad, presentación clínica: debut o recurrencia; año del diagnóstico, SG y subtipo tumoral. La SG la definimos como el tiempo desde el diagnóstico del CM hasta la muerte del paciente por cualquier causa.

El subtipo tumoral lo definimos como luminal B HER2+ en aquellos tumores con expresión de receptores de estrógenos y/o progesterona además de HER2+; por otra parte, HER2 enriquecidos se consideró a aquellos tumores con sobreexpresión o amplificación aislada de HER2 sin expresión de receptores hormonales. La positividad de HER2 se definió como la expresión de HER2 por inmunohistoquímica (IHQ) definida como 3+, o la amplificación del gen de HER2 estudiada a través de hibridación in situ con inmunofluorescencia (FISH) en casos de IHQ 2+.

En relación al tratamiento, analizamos nuestra cohorte según el año de diagnóstico, dividiéndola en 2 períodos (1997-2006 y 20072017), con objeto de dar cuenta del uso de nuevas terapias y estrategias de manejo en la última década.

Para el análisis de los datos utilizamos estadística descriptiva, la SG la calculamos con el método de Kaplan Meier y comparamos las curvas de SG a través del test de Log Rank. Utilizamos el programa estadístico XLSTAT v.19.4.

\section{Resultados}

Del total de pacientes ingresados en nuestra base de datos (2.724), tenemos información del subtipo en 2.149 (79\%). De estos, 18\% correspondía a tumores HER2+ (LB-HER2+ y HER enriquecidos; 395/2.149) (Figura 1). Disponemos de información de la etapa en 368 de 395 pacientes; de ellos, $43(11,6 \%)$ correspondía a etapas IV al debut, con predominio, en este último grupo, de

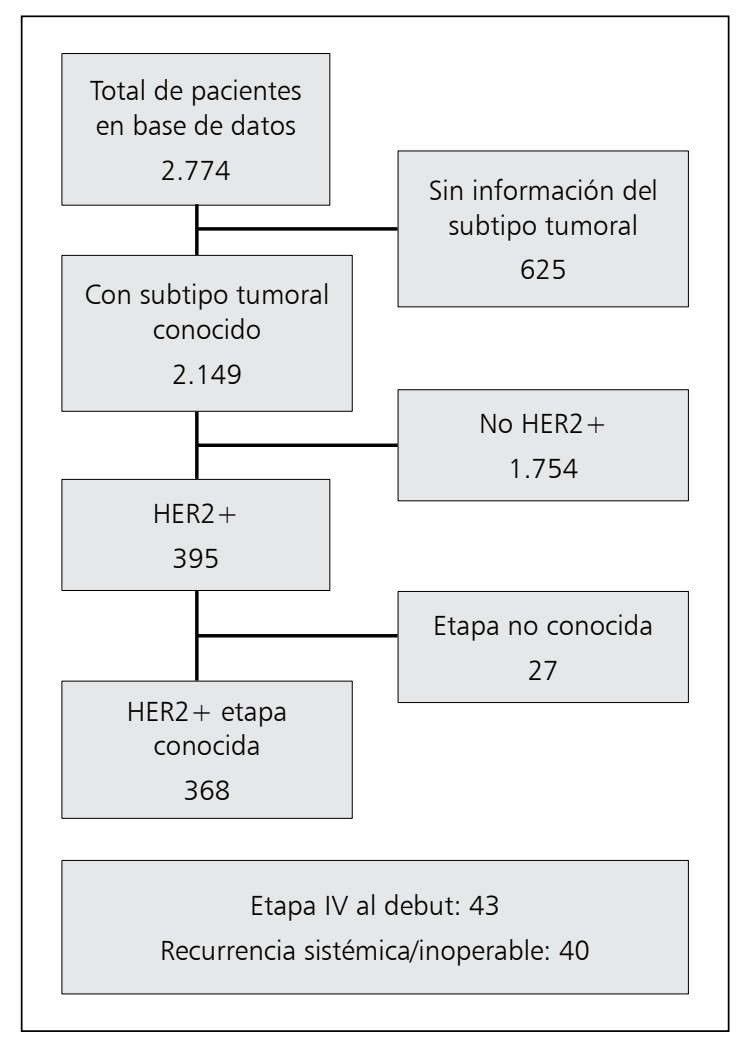

Figura 1. Flujograma de análisis de pacientes en base datos. 
tumores HER2 enriquecidos (53,4\%). Cuarenta pacientes $(10,8 \%)$ han tenido una recurrencia sistémica o inoperable de la enfermedad; por tanto, existen 83 pacientes con CM avanzado HER2+ que constituyó la muestra analizada en este estudio.

Las características de los pacientes se describen en la Tabla 1. Casi dos tercios de los pacientes fueron tratados después de 2007, con una tendencia a mayor edad en este último grupo, no estadísticamente significativa. Más pacientes diagnosticados entre los años 2007 y 2017 fueron diagnosticadas con etapa IV al debut (14\% entre los años 19972006 y $86 \%$ los años 2007-2017, p = 0,001).

La mediana de SG fue de 24 meses (Figura 2).
No hubo diferencia estadísticamente significativa al comparar la SG de pacientes con CM HER2+ etapa IV (al debut) respecto de aquellos que sufrieron recurrencia de la enfermedad (Figura 3).

Al comparar pacientes con $\mathrm{CM}$ avanzado HER2+ diagnosticados entre los años 1997 y 2006 y aquellos con diagnóstico entre los años 2007 y 2017, encontramos en este último grupo que fue más frecuente la presentación en etapa IV al debut, y la mediana de SG fue casi el doble de aquella estimada para pacientes con diagnóstico de $\mathrm{CM}$ entre los años 1997-2006 (17 vs 32 meses, respectivamente, Figura 4); sin embargo, esta diferencia no es estadísticamente significativa $(\mathrm{p}=0,09)$.

Tabla 1. Características de pacientes con cáncer de mama avanzado HER2+

\begin{tabular}{|c|c|c|c|c|}
\hline & $1997-2006(n / \%)$ & $2007-2017(n / \%)$ & $\mathbf{P}$ & Total \\
\hline Número de pacientes & $25(30,1 \%)$ & $58(69,9 \%)$ & & 83 \\
\hline Edad (mediana, rango) & $46(24-77)$ & $52,5(30-88)$ & 0,32 & 55 \\
\hline $\begin{array}{l}\text { Presentación } \\
\text { IV (debut) } \\
\text { Recurrencias }\end{array}$ & $\begin{array}{r}6(14,0 \%) \\
19(47,5 \%)\end{array}$ & $\begin{array}{l}37(86,0 \%) \\
21(52,5 \%)\end{array}$ & 0,001 & $\begin{array}{l}43(51,8 \%) \\
40(49,2 \%)\end{array}$ \\
\hline Mediana seguimiento (meses) & 62 & 25 & 0,13 & \\
\hline Mediana sobrevida global (meses) & 17 & 32 & 0,09 & \\
\hline
\end{tabular}

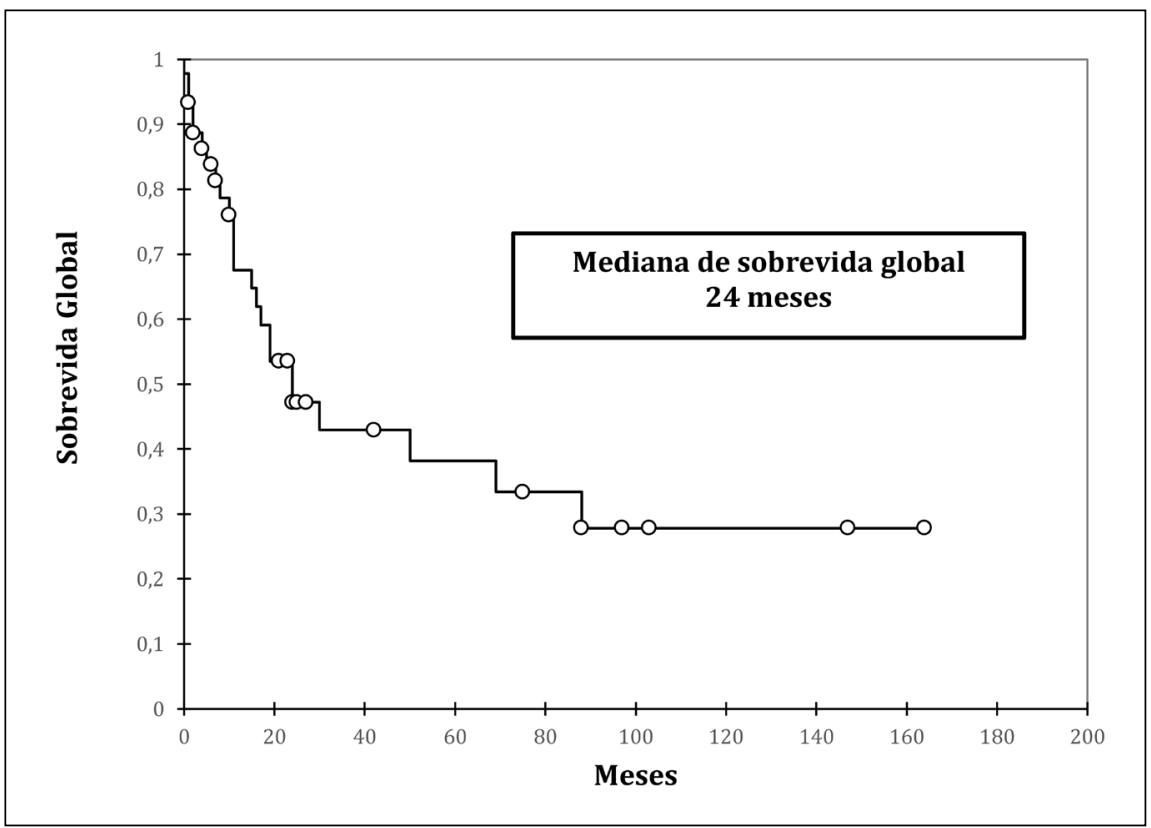

Figura 2. Sobrevida global de pacientes con cáncer de mama avanzado HER2 + . 


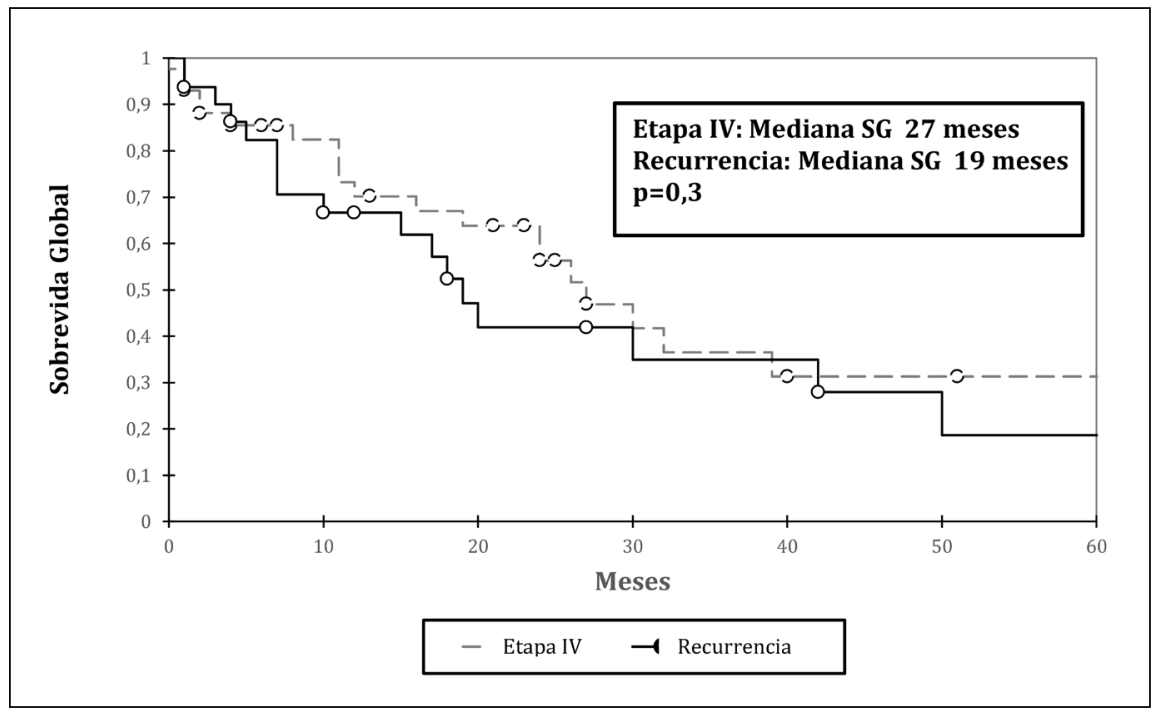

Figura 3. Sobrevida global de pacientes con cáncer de mama avanzado HER2+. Etapa IV versus recurrencia. SG: Sobrevida global.

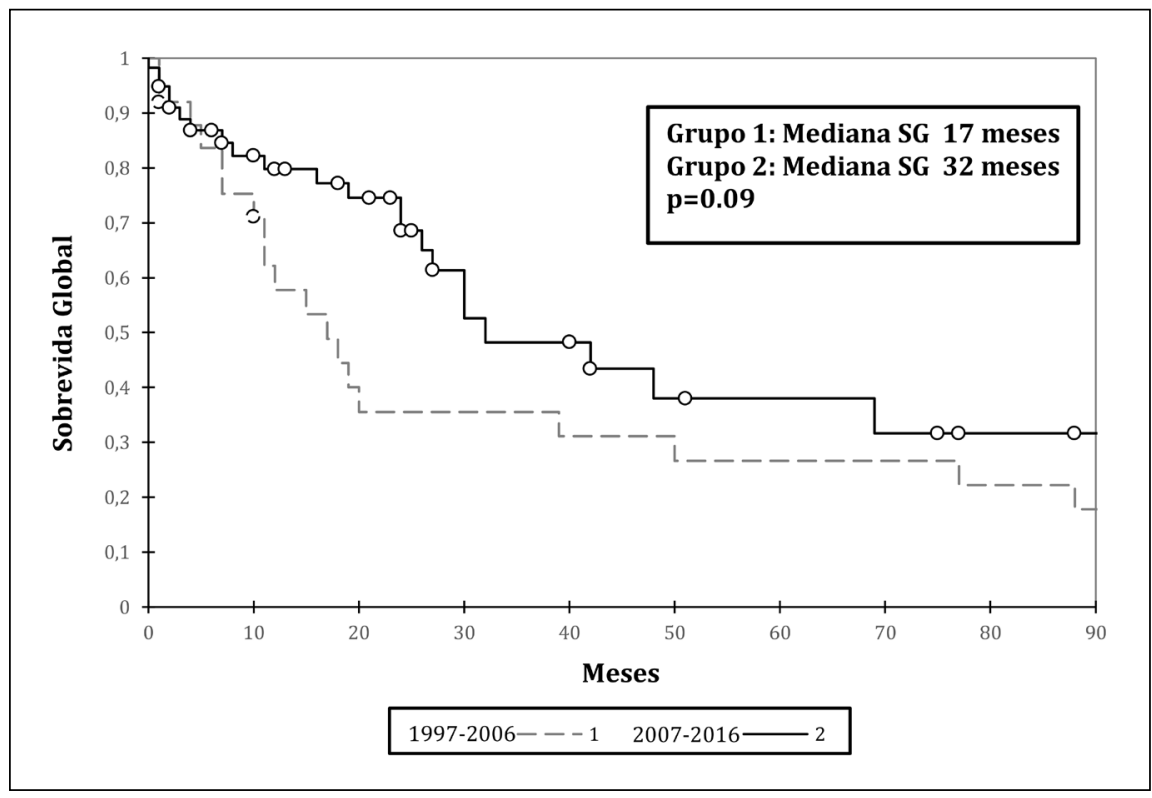

Figura 4. Sobrevida global de pacientes con cáncer de mama avanzado según año al diagnóstico. SG: Sobrevida global.

\section{Discusión}

La SG de pacientes con CM avanzado HER2+ ha mejorado en los últimos diez años en nuestro centro, al igual como ha sucedido alrededor del mundo ${ }^{8,9}$. La identificación de este subtipo tumoral y mejores opciones de tratamiento explican estas cifras (Figura 4, Tabla 2).

A pesar de que la mediana de SG reportada de nuestros pacientes con CM HER2+ avanzado parece similar a la publicada en el estudio original de Slamon que utilizó trastuzumab más quimioterapia $^{10}$ (24 meses en nuestro estudio vs 25 meses en el estudio de Slamon), es muy inferior a la publicada en un estudio posterior llamado Cleopatra, que usa un bloqueo dual de HER2 con trastuzumab y pertuzumab ${ }^{6}$ (56 meses) (Tabla 2 ). Un factor que puede explicar la diferencia en 
Tabla 2. Principales estudios fase 3 publicados que sustentan evidencia de terapia anti-HER2 estándar en cáncer de mama avanzado HER2+

\begin{tabular}{|c|c|c|c|c|c|c|}
\hline $\begin{array}{l}\text { Terapia } \\
\text { anti-HER2 }\end{array}$ & $\begin{array}{l}\text { Revista, año } \\
\text { publicación }\end{array}$ & $\mathbf{n}$ & Ramas & $\begin{array}{l}\text { Objetivo } \\
\text { primario }\end{array}$ & $\begin{array}{l}\text { Resultados } \\
\text { (meses) }\end{array}$ & $\begin{array}{l}\text { Mediana } \\
\text { SG (meses) }\end{array}$ \\
\hline Trastuzumab & NEJM, 2001 & 469 & $\begin{array}{l}\text { 1: Trastuzumab + QT } \\
\text { 2: QT }\end{array}$ & SLP & $\begin{array}{l}1: 7,4 \\
2: 4,6\end{array}$ & $\begin{array}{l}1: 25,1 \\
2: 20,3\end{array}$ \\
\hline Lapatinib & $\begin{array}{l}\text { NEJM, } 2006 \\
\text { BCRT, } 2008\end{array}$ & 399 & $\begin{array}{l}\text { 1: Lapatinib + QT } \\
\text { 2: QT }\end{array}$ & SLP & $\begin{array}{l}1: 6,2 \\
2: 4,3\end{array}$ & $\begin{array}{l}1: 15,6 \\
2: 15,3\end{array}$ \\
\hline $\begin{array}{l}\text { Pertuzumab }+ \\
\text { trastuzumab }\end{array}$ & $\begin{array}{l}\text { NEJM, } 2012 \\
\text { NEJM, } 2015\end{array}$ & 808 & $\begin{array}{l}\text { 1: Trastuzumab + pertuzumab + QT } \\
\text { 2: Trastuzumab + QT }\end{array}$ & SLP & $\begin{array}{l}1: 12,4 \\
2: 18,5\end{array}$ & $\begin{array}{l}1: 56,5 \\
2: 40,8\end{array}$ \\
\hline TDM1* & NEJM, 2012 & 991 & $\begin{array}{l}\text { 1: TDM1 } \\
\text { 2: Lapatinib + QT }\end{array}$ & SLP/SG & $\begin{array}{l}1: 9,6 \\
2: 6,4\end{array}$ & $\begin{array}{l}1: 30,9 \\
2: 25,1\end{array}$ \\
\hline
\end{tabular}

*Pacientes previamente tratados con trastuzumab. QT: quimioterapia. SLP: sobrevida libre de progresión. SG: Sobrevida global.

SG entre lo reportado por este último trabajo y nuestra población son las características de los pacientes incluidos en ambos estudios clínicos, como es habitual para la población seleccionada en estudios fase $3^{11}$ : buena capacidad funcional, exámenes de laboratorio aceptables para ingresar al estudio y ausencia de metástasis en sistema nervioso central, entre otros; estas características difieren de pacientes tratados en la práctica clínica habitual, que no cumplen los estrictos criterios de ingreso a dichos estudios controlados, como son los reportados en este trabajo. Además, no todos los pacientes diagnosticados en el último período analizado recibieron la combinación de anticuerpos (Ac) anti-HER2.

Un hallazgo llamativo en el análisis de nuestros datos es la mayor presentación de CM etapa IV en el último decenio ( $14 \%$ vs $86 \%)$. Una explicación potencial puede ser la mejor etapificación de pacientes en los últimos años, dada la incorporación de mejores métodos diagnósticos por imágenes, por ejemplo, el uso de tomografía computada con emisión de positrones (FISH) o de resonancia magnética. Este cambio también podría explicar indirectamente el mejor pronóstico en neoplasias en etapa IV, dada la probabilidad de encontrar más enfermedad oligometastásica o enfermedad metastásica indolente de buen pronóstico, y que con otros métodos diagnósticos pudiese no haber sido diagnosticada ${ }^{12}$.

La evolución del tratamiento del CM HER2+ ha mostrado gran dinamismo en las últimas décadas. Mejorías en los métodos diagnósticos para tipificar la enfermedad, en esquemas de tra- tamientos y cuidados de soporte podrían explicar un aumento sostenido en los niveles de sobrevida del CM avanzado HER2 $+^{8,12-16}$. Al tratamiento citotóxico clásico inicial se han agregado la combinación con terapias biológicas en base a un Ac monoclonal anti-HER2 llamado trastuzumab, un inhibidor de tirosina kinasa de HER2 (lapatinib), los que inhiben la acción proliferativa del HER2 ${ }^{15,17}$; y la aparición de inmunoconjugados, que combinan quimioterapia y trastuzumab en la misma molécula ${ }^{18}$. Recientemente, la adición de otro Ac anti-HER2, llamado pertuzumab, ha demostrado mejorar los resultados de SG en CM avanzado, siendo, actualmente, la combinación de ambos anticuerpos en conjunto con quimioterapia, el tratamiento estándar para el CM avanzado HER $2+{ }^{6}$. Estos cambios se han reflejado también en nuestro país.

El Ministerio de Salud de Chile, en la guía de práctica clínica para $\mathrm{CM}$, realiza recomendaciones relacionadas con el uso de Ac anti-HER2. A medida que se han desarrollado nuevas terapias biológicas, se han incluido en las actualizaciones. El año 2016 entra en vigencia la Ley 20.850 que subsidia el Ac trastuzumab, permitiendo el acceso universal a esta terapia para pacientes con $\mathrm{CM}$ HER2+ (Figura 5).

Mientras creemos que la aparición, disponibilidad y acceso de nuevas terapias explican la mejor SG vista en la última década en nuestros pacientes, la naturaleza retrospectiva de nuestros datos puede afectar esta conclusión. Sesgos en la selección de pacientes, métodos diagnósticos y desconocimiento de terapias antineoplásicas y de 


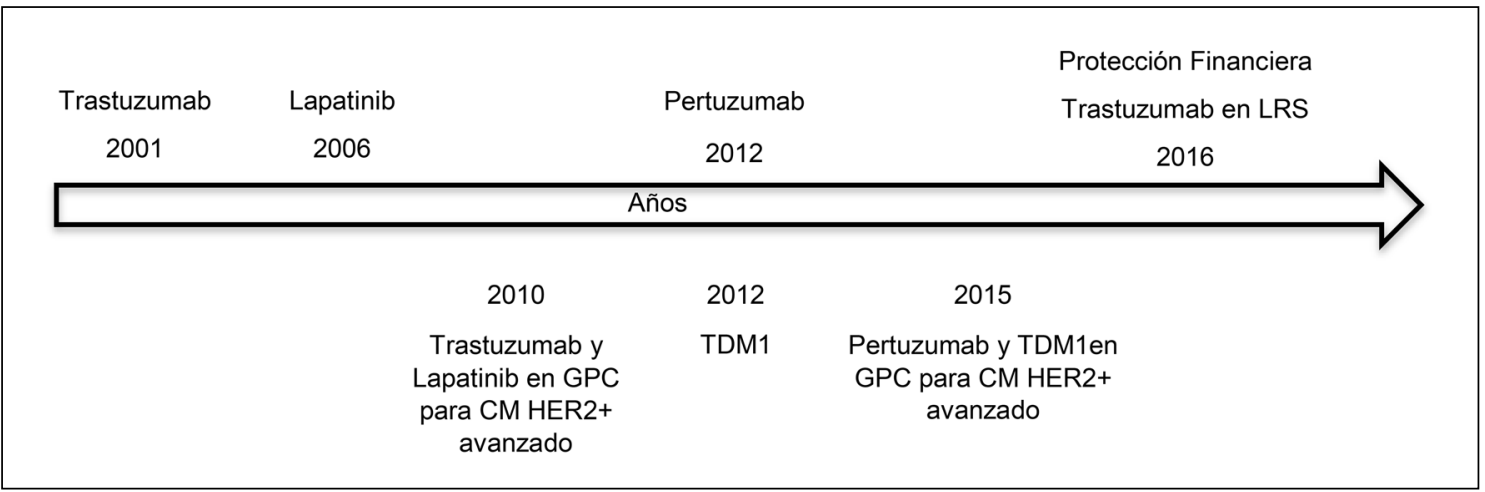

Figura 5. Línea de tiempo de desarrollo de principales agentes anti-HER2 usados en la práctica clínica para cáncer de mama avanzado HER2 + y recomendaciones del uso de estos en guías de práctica clínica del Ministerio de Salud de Chile. LRS: Ley 20.850 Ricarte Soto. GPC: Guía práctica clínica del Ministerio de Salud de Chile. CM: Cáncer de mama.

soporte en nuestra serie son factores a considerar. Nuestro tamaño muestral limitado impide demostrar, por ahora, que la diferencia en SG, clínicamente relevante entre ambos períodos estudiados, es estadísticamente significativa.

Conocer el subtipo del CM es relevante para predecir su historia natural y, además, determinar tratamientos específicos y personalizados. Particularmente para CM avanzado HER2+, la aparición de nuevas y eficaces terapias ha duplicado su sobrevida, logrando incluso, en algunos pacientes, estabilidad a largo plazo de la enfermedad, convirtiendo a esta patología en una enfermedad crónica para algunas personas ${ }^{19}$. Mejorar los métodos diagnósticos, particularmente la identificación del subtipo tumoral por técnicas de inmunohistoquímica o a través de FISH, y asegurar acceso oportuno y universal a estas terapias, debe ser un objetivo permanente.

\section{Referencias}

1. Estadísticas Vitales. Instituto Nacional de Estadísticas. INE 2014. Disponible en: http://www.ine.cl/estadisticas/ demograficas-y-vitales [Consultado el 11 de abril de 2018].

2. Goldhirsch A, Wood WC, Coates AS, Gelber RD, Thürlimann B, Senn H-J. Strategies for subtypes--dealing with the diversity of breast cancer: highlights of the St. Gallen International Expert Consensus on the Primary Therapy of Early Breast Cancer 2011. Ann Oncol 2011; 22 (8): 1736-47.
3. Pan H, Gray R, Braybrooke J, Davies C, Taylor C, McGale P, et al. 20-Year Risks of Breast-Cancer Recurrence after Stopping Endocrine Therapy at 5 Years. N Engl J Med 2017; 377 (19): 1836-46.

4. Cardoso F, Costa A, Senkus E, Aapro M, André F, Barrios $\mathrm{CH}$, et al. 3rd ESO-ESMO International Consensus Guidelines for Advanced Breast Cancer (ABC 3). Ann Oncol 2017; 28 (1): 16-33.

5. Wolff AC, Hammond MEH, Hicks DG, Dowsett M, McShane LM, Allison $\mathrm{KH}$, et al. Recommendations for human epidermal growth factor receptor 2 testing in breast cancer: American Society of Clinical Oncology/ College of American Pathologists clinical practice guideline update. J Clin Oncol 2013; 31 (31): 3997-4013.

6. Swain SM, Kim S-B, Cortés J, Ro J, Semiglazov V, Campone $\mathrm{M}$, et al. Pertuzumab, trastuzumab, and docetaxel for HER2-positive metastatic breast cancer (CLEOPATRA study): overall survival results from a randomised, double-blind, placebo-controlled, phase 3 study. Lancet Oncol 2013; 14 (6): 461-71.

7. MINSAL. Guías Clínicas AUGE, Cancer de mama. 2015. Disponible en: http://www.minsal.cl/wp-content/ uploads/2015/09/GPC-CaMama.pdf [Consultado el 11 de abril de 2018].

8. Slamon D, Eiermann W, Robert N, Pienkowski T, Martin M, Press M, et al. Adjuvant Trastuzumab in HER2-Positive Breast Cancer. N Engl J Med 2011; 365 (14): 1273-83.

9. Chen TW, Lin C, Huang C. Should pertuzumab be used as part of neoadjuvant treatment prior to the release of the APHINITY trial results? Transl Cancer Res 2016; 5 (S4): S907-12.

10. Slamon DJ, Leyland-Jones B, Shak S, Fuchs H, Paton V, 
Bajamonde A, et al. Use of Chemotherapy plus a Monoclonal Antibody against HER2 for Metastatic Breast Cancer That Overexpresses HER2. N Engl J Med 2001; 344 (11): 783-92.

11. Lichtman SM, Harvey RD, Damiette Smit M-A, Rahman A, Thompson MA, Roach N, et al. Modernizing Clinical Trial Eligibility Criteria: Recommendations of the American Society of Clinical Oncology-Friends of Cancer Research Organ Dysfunction, Prior or Concurrent Malignancy, and Comorbidities Working Group. J Clin Oncol 2017; 35 (33): 3753-9.

12. Di Lascio S, Pagani O. Oligometastatic Breast Cancer: A Shift from Palliative to Potentially Curative Treatment? Breast Care 2014; 9 (1): 7-14.

13. Jerusalem G, Bachelot T, Barrios C, Neven P, Di Leo A, Janni W, et al. A new era of improving progression-free survival with dual blockade in postmenopausal HR+, HER2-advanced breast cancer. Cancer Treat Rev 2015; 41 (2): 94-104.

14. Coates AS, Winer EP, Goldhirsch A, Gelber RD, Gnant $\mathrm{M}$, Piccart-Gebhart $\mathrm{M}$, et al. Tailoring therapies--improving the management of early breast cancer: St Gallen International Expert Consensus on the Primary Therapy of Early Breast Cancer 2015. Ann Oncol 2015; 26 (8): 1533-46.

15. Zardavas D, Cameron D, Krop I, Piccart M. Beyond Trastuzumab and Lapatinib: New Options for HER2-Positive Breast Cancer. Am Soc Clin Oncol Educ Book. 2013.

16. Puglisi F, Fontanella C, Amoroso V, Bianchi GV, Bisagni G, Falci C, et al. Current challenges in HER2-positive breast cancer. Crit Rev Oncol Hematol 2016; 98: 211-21.

17. Geyer CE, Forster J, Lindquist D, Chan S, Romieu CG, Pienkowski T, et al. Lapatinib plus Capecitabine for HER2-Positive Advanced Breast Cancer. N Engl J Med 2006; 355 (26): 2733-43.

18. Verma S, Miles D, Gianni L, Krop IE, Welslau M, Baselga J, et al. Trastuzumab Emtansine for HER2-Positive Advanced Breast Cancer. N Engl J Med 2012; 367 (19): 1783-91.

19. Metzger-Filho O, Sun Z, Viale G, Price KN, Crivellari D, Snyder RD, et al. Patterns of Recurrence and Outcome According to Breast Cancer Subtypes in Lymph Node-Negative Disease: Results From International Breast Cancer Study Group Trials VIII and IX. J Clin Oncol 2013; 31 (25): 3083-90. 